\title{
Uma contribuição ao auxílio do diagnóstico do autismo a partir do processamento de imagens para extração de medidas antropométricas
}

\section{A contribution to aid the diagnosis of autism from image processing to extract anthropometric measurements}

\author{
Samuel Portela de Carvalho ${ }^{1,2}$ \\ Ariane Machado Lima ${ }^{1,3}$ \\ Helena Brentani ${ }^{4,5}$ \\ Rodrigo Ambrósio Fock ${ }^{6,6}$ \\ Décio Brunoni 7,8 \\ Fátima L. S. Nunes ${ }^{1,9}$
}

Data de submissão: 23/11/2015, Data de aceite: 24/09/2016

\footnotetext{
${ }^{1}$ Laboratório de Aplicações de Informática em Saúde. Escola de Artes, Ciências e Humanidades - Universidade de São Paulo. Av. Arlindo Béttio, 1000, Ermelino Matarazzo, São Paulo-SP.

2 \{samuel.portela.carvalho@usp.br\}

3 ariane.machado@usp.br\}

${ }_{4}^{4}$ Instituto de Psiquiatria, Faculdade de Medicina - Universidade de São Paulo. Rua Dr. Ovídio Pires de Campos, 785, São Paulo-SP.

5 helena.brentani@gmail.com $\}$

6 \{rodrigo.fockegmail.com\}

${ }^{7}$ Programa de Pós-Graduação em Distúrbios do Desenvolvimento - Universidade Presbiteriana Mackenzie - Rua da Consolação 930, Prédio 28, São Paulo-SP.

${ }^{8}$ \{debruno46@gmail.com\}

9 \{fatima.nunes@usp.br\}
} 
Uma contribuição ao auxílio do diagnóstico do autismo a partir do processamento de imagens para extração de medidas antropométricas

Resumo: O Transtorno do Espectro Autista (TEA) é uma síndrome caracterizada pela dificuldade na interação social e desvios qualitativos na comunicação e no uso da imaginação. Embora o diagnóstico dessa síndrome consista basicamente de avaliações clínicas, há indícios de que crianças portadoras de TEA também apresentem medidas antropométricas faciais diferentes das crianças sem a síndrome. Neste caso, ferramentas computacionais podem ser empregadas como coadjuvantes nesta tarefa. O presente trabalho tem o objetivo de definir e validar técnicas para processar imagens faciais e medir distâncias antropométricas com a finalidade de auxiliar o diagnóstico de TEA. As técnicas definidas culminaram na construção de uma ferramenta computacional capaz de analisar imagens de pacientes e calcular as medidas antropométricas faciais. A ferramenta foi validada com um banco de imagens de indivíduos com e sem a síndrome, tendo sido encontradas semelhanças e diferenças entre as medidas antropométricas extraídas pela ferramenta e medidas citadas em estudos anteriores. A ferramenta mostrou-se capaz de processar imagens frontais de pacientes e extrair seus componentes e medidas antropométricas com precisão, a partir de técnicas de processamento de imagens adaptadas para essas finalidades, podendo contribuir efetivamente para o auxílio ao diagnóstico de TEA.

Palavras-chave: transtorno do espectro autista, TEA, imagens faciais, antropometria, autismo

Abstract: Autism Spectrum Disorder (ASD) is a syndrome characterized by difficulties in social interaction and qualitative problems in communication and use of imagination. Although the diagnosis of this syndrome consists primarily of clinical evaluation, there is evidence that children with ASD have different anthropometric measures when compared to children without the syndrome. However, obtaining and comparing these measures is not trivial, and computer tools can be used to support this task. This study aims to define and validate techniques for processing facial images and measuring anthropometric distances to aid the diagnosis of ASD. The defined techniques culminated in the construction of a computational tool able to analyze images of patients and calculate the facial anthropometric measures. The tool was validated using an image database of individuals with and without the syndrome, and similarities and differences between the anthropometric measures taken by the tool and measures mentioned in previous studies were found. The tool proved to be capable of processing patients' frontal images, as well as extracting their components and anthropometric measures accurately using techniques adapted for these purposes. Thus, it can effectively contribute to aid the diagnosis of ASD.

Keywords: autistic spectrum disorder, ASD, facial images, anthropometry, autism 
Uma contribuição ao auxílio do diagnóstico do autismo a partir do processamento de imagens para extração de medidas antropométricas

\section{Introdução}

O Transtorno do Espectro Autista (TEA) é uma síndrome caracterizada pela dificuldade na interação social e desvios qualitativos na comunicação e no uso da imaginação [1]. A síndrome pode ser definida também como "perda de contato com a realidade, causada pela impossibilidade ou grande dificuldade na comunicação interpessoal" [2]. Leo Kanner, em 1943, distinguiu o autismo de outras psicoses graves da infância, apresentando dois critérios principais que definiriam o autismo como "a solidão e a insistência obsessiva na invariância" [2].

Hans Asperger, em 1944, referindo-se ao mesmo tipo de crianças descritas por Kanner, denominou o quadro de psicopatologia autística. Asperger descreveu os distúrbios comportamentais das crianças por ele investigadas, resumido-os em: "compulsividade e obsessão de pensamentos e ações, linguagem de expressão bizarra, e limitação das relações sociais, que comprometem sua personalidade" [3].

O diagnóstico da síndrome é realizado com ferramentas específicas. Segundo [3], é necessária uma análise detalhada com avaliação clínica, laboratorial e genética, bem como o uso de checklists de comportamentos autísticos, complementando os critérios estabelecidos nas classificações internacionais.

Consoantes com [3], [4] afirmam que os critérios utilizados para diagnosticar o autismo são aqueles descritos no Manual Estatístico e Diagnóstico da Associação Americana de Psiquiatria - DSM - e que os exames laboratoriais continuam sendo imprescindíveis. Já [1] traz uma descrição oposta, dizendo que o diagnóstico do autismo é feito basicamente através de avaliação clínica e não existem testes laboratoriais específicos para a detecção da síndrome.

Algumas iniciativas têm utilizado a tecnologia para auxiliar o diagnóstico, como [5], que desenvolveram um software de processamento de imagens e visão computacional para aumentar a exatidão na triagem de crianças submetidas ao diagnóstico de autismo a partir da análise de vídeos de testes comportamentais. Recentemente, alguns pesquisadores têm concentrado esforços na verificação da relação do autismo com a Antropometria, que é a "ciência que estuda as medidas de tamanho, peso e proporções do corpo humano a partir de medições de uma população estudada" [6].

Em [7] destaca-se uma tendência ao aumento no peso e no Índice de Massa Corporal (IMC), bem como nas medidas das pregas cutâneas do tríceps, subescapular e suprailíaca em crianças autistas, se comparadas a crianças normais. Em [8], 65 meninos de 8 a 12 anos com TEA e 41 meninos com desenvolvimento normal tiveram seus fenótipos faciais comparados, por meio da extração de pontos de referência do rosto. Os resultados mostraram que meninos com TEA possuíam um fenótipo facial distinto dos meninos com desenvolvimento normal. 
Uma contribuição ao auxílio do diagnóstico do autismo a partir do processamento de imagens para extração de medidas antropométricas

Ademais, [9], estudando Minor Physical Anomalies (MPAs) concluíram que pessoas adultas com TEA apresentaram maiores taxas de MPAs quando comparadas a um grupo de controle.

Apesar da existência de estudos que relacionam medidas antropométricas com diagnóstico de TEA, não foram encontrados na literatura trabalhos dedicados a desenvolver técnicas e ferramentas computacionais que favoreçam esta abordagem. Com a finalidade de contribuir com o preenchimento desta lacuna, o presente trabalho tem o objetivo de definir e validar um conjunto de técnicas de processamento de imagens adaptadas para extrair medidas antropométricas faciais de imagens frontais de indivíduos. A ferramenta resultante dessas técnicas foi validada com dois grupos distintos de imagens, um composto por pacientes com TEA e outro composto por indivíduos sem TEA. O diferencial deste trabalho é a extração automática dos pontos de referência faciais (landmarks), sem a necessidade de utilização de outros programas de processamento de imagens ou intervenção manual do usuário.

Além desta introdução, este documento é composto por mais cinco seções. A seção 3 aborda aspectos conceituais que fundamentam este trabalho; a seção 3 apresenta os trabalhos relacionados ao tema; a seção 4 explica os métodos e técnicas utilizadas para construir e validar a ferramenta; a seção 5 contém os resultados encontrados e uma discussão sobre eles; por fim, a seção 6 traz as conclusões e algumas considerações finais.

\section{Aspectos conceituais}

\subsection{Processamento de imagens digitais}

Uma imagem, em sua representação digital, é uma função $f(x, y)$, em que $x$ e $y$ representam as coordenadas espaciais dos pontos que formam a imagem e o valor de $f(x, y)$ é proporcional ao brilho ou cor da imagem naquele ponto. Como a luz é uma forma de energia, $f(x, y)$ deve ser positiva e finita [10]. Dessa forma, uma imagem digital no domínio espacial pode ser considerada como uma matriz cujos índices de linhas e colunas representam um determinado ponto na imagem e o valor correspondente do elemento na matriz identifica a cor naquele ponto. A cada um desses pontos é dado o nome de pixel.

No processamento de imagens, são aplicados algoritmos e/ou funções matemáticas nas matrizes que representam as imagens digitais, com a finalidade de solucionar algum problema específico. Podem ser feitos ajustes de brilho e contraste, recortes, aplicação ou eliminação de ruídos, etc [10].

\subsection{Antropometria}

A Antropometria - do grego anthropos, humano e metron, medida [11] - é a ciência que estuda o corpo humano por meio da análise quantitativa, considerando medidas de ta- 
Uma contribuição ao auxílio do diagnóstico do autismo a partir do processamento de imagens para extração de medidas antropométricas

manho, peso e proporções [6]. Na Medicina, a Antropometria é empregada de diversas formas e para diversos fins. Segundo [12], medidas antropométricas ajudam no planejamento e avaliação de cirurgias plásticas e reconstrutivas. [13] discute o uso da Antropometria como uma técnica de medida da composição corporal, útil para pesquisas básicas de caráter experimental e epidemiológico, diagnóstico e controle de patologias e planejamento de dietas e programas para controle de peso corporal.

A Antropometria facial "é um método de análise não invasivo, que pode ser direto, obtido por meio da medição na própria pessoa estudada, ou indireto, a partir de radiografias e fotografias" [14]. Para essa medição, são utilizados pontos bem definidos na face, chamados landmarks. A Figura 1 ilustra esses pontos, nomeados de acordo com a nomenclatura anatômica grega ou latina por [6].

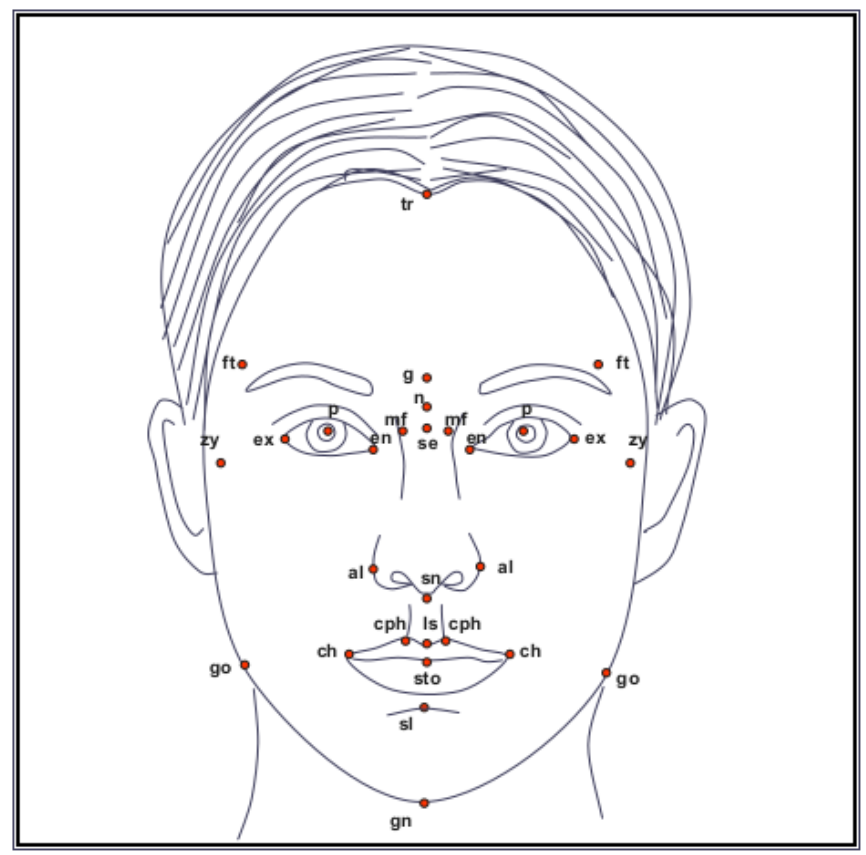

Figura 1. Landmarks faciais frontais [11]. 
Uma contribuição ao auxílio do diagnóstico do autismo a partir do processamento de imagens para extração de medidas antropométricas

\section{Trabalhos Relacionados}

Alguns trabalhos na literatura especializada da área apontam diferenças nas medidas antropométricas entre portadores e não portadores de TEA. Essas diferenças existem em praticamente todo o corpo, incluindo tamanho das mãos, tamanho dos pés, circunferência da cabeça, peso, altura e Índice de Massa Corporal (IMC) [9], [7], [15]. Outros trabalhos utilizam ferramentas computacionais para auxiliar o diagnótico a partir da análise do comportamento do indivíduo, como mostrado a seguir.

Usando a computação no auxílio ao diagnóstico do autismo, [5] desenvolveram um programa de visão computacional que analisa quadros de vídeos de crianças em testes comportamentais. Esses testes servem para detectar transtornos de comportamento, o que pode melhorar a exatidão do diagnóstico. O programa processa os quadros dos vídeos adquiridos e detecta as reações das crianças a cada tarefa executada, baseado nos movimentos dos olhos, do rosto, dos braços e do tronco.

De acordo com [8], o cérebro se desenvolve em sintonia e em coordenação com o desenvolvimento dos tecidos faciais. O TEA resulta de alterações no cérebro embriológico, fazendo com que as faces de crianças com a síndrome apresentem pequenas diferenças se comparadas com crianças com desenvolvimento normal. Os pesquisadores estudaram 105 meninos entre 8 e 12 anos, dos quais 64 eram portadores de TEA. Essas crianças foram fotografadas de vários ângulos diferentes e as imagens tridimensionais das suas faces foram geradas a partir do software $3 d M D ®$ Cranial System e as coordenadas tridimensionais de 17 landmarks foram coletadas por dois classificadores, utilizando o software 3dMDpatient $\mathrm{R}$. Em seguida, foi gerada uma matriz de distâncias Euclidianas entre todos os pares de landmarks possíveis. Os valores foram estatisticamente analisados e dois resultados importantes foram obtidos:

1. Meninos diagnosticados com TEA demonstraram diferenças significativas na morfologia facial se comparados aos meninos sem TEA. As seguintes distâncias são reduzidas no grupo de meninos com TEA: g-en, n-en, n-al, n-sn, n-cph, e n-ls. Da mesma forma, as seguintes distâncias são maiores no grupo de meninos com TEA: ch-al, ch-ex, al-ex, ch-ch, ch-cph, ch-ls, ch-gn, cph-sn, ex-en, ft-en, ft-al, ft-che e ft-ex.

2. Os meninos diagnosticados com TEA apresentaram características antropométricas diferentes entre si, dividindo-os em dois subgrupos. No subgrupo 1, as seguintes medidas são reduzidas em comparação ao outro: ft-ch, ft-al, ft-sn, ft-ex, ft-n e ft-en. Já no subgrupo 2, as seguintes medidas são maiores em comparação ao subgrupo 1: ft-ch, ex-ch, ex-cph, en-al, en-sn e g-al.

Em resumo, os meninos com TEA apresentaram medidas maiores na largura da boca, das órbitas e da parte superior da face, combinado com o achatamento da ponte nasal e altura 
Uma contribuição ao auxílio do diagnóstico do autismo a partir do processamento de imagens para extração de medidas antropométricas

reduzida do filtro e da região maxilar, além de um estreitamento na distância entre os cantos dos olhos.

Com base nos resultados obtidos em [8], em [16] foram usadas as medidas antropométricas faciais de 62 crianças com idades entre 8 e 12 anos diagnosticadas com TEA para classificá-las em diferentes grupos de acordo com as características antropométricas. A diferença deste trabalho para o anterior é o emprego de distâncias geodésicas - distâncias obtidas medindo-se a superfície tridimensional do rosto, considerando-se os relevos e depressões dos componentes faciais [16] - ao invés de distâncias Euclidianas entre os landmarks considerados. Além das 62 crianças com TEA, participaram também desse estudo 36 crianças com desenvolvimento normal (grupo de controle), todas com idades entre 8 e 12 anos. Para a aquisição dos landmarks e das medidas entre eles, foi utilizado o software $3 d M D \cap C r a-$ nial System - o mesmo utilizado por [8] - que constrói um modelo tridimensional (3D) dos rostos das crianças estudadas. Foram considerados 19 landmarks e as distâncias geodésicas entre todos os possíveis pares de landmarks foram computadas, obtendo-se um total de 171 distâncias. A Tabela 1 mostra a relação entre os landmarks apresentados na Figura 1 e aqueles que foram considerados por [8] e [16].

\section{Métodos}

O cálculo das medidas antropométricas realizado neste trabalho compreende a detecção da face e dos componentes faciais (olhos, nariz e boca) e a localização de 17 pontos (landmarks) faciais - os mesmos utilizados por [8] - por meio da aplicação de técnicas de processamento de imagens. Após a detecção dos componentes e a localização desses pontos, as 136 distâncias Euclidianas entre todos os pares distintos possíveis dentre os 17 landmarks $\left(C_{17,2}=136\right)$ são calculadas para gerar as medidas. É importante salientar a diferença deste trabalho para os dois trabalhos citados anteriormente, [8] e [16]. Nos trabalhos anteriores foram utilizadas representações tridimensionais das faces dos indivíduos e foram calculadas distâncias geodésicas entre os landmarks. Já no presente trabalho, foram utilizadas imagens planas e distâncias Euclidianas entre os landmarks. A ferramenta resultante das técnicas desenvolvidas foi validada com grupos de indivíduos com e sem TEA, como mostrado a seguir.

\subsection{Detecção da face e dos componentes faciais}

Para a detecção da face, foi utilizado o algoritmo de detecção Viola-Jones, por meio de classificadores Haar Cascade [17], disponível na biblioteca OpenCV [18]. Este algoritmo tenta encontrar em uma imagem características que codificam alguma informação da classe desejada (no caso, a face). A face detectada é marcada por um retângulo, conforme mostrado na Figura 2. 
Uma contribuição ao auxílio do diagnóstico do autismo a partir do processamento de imagens para extração de medidas antropométricas

Tabela 1. Landmarks faciais apresentados na Figura 1 e considerados em estudos anteriores

\begin{tabular}{|c|c|c|c|}
\hline & Landmarks & (ALDRIDGE et al, 2011) & (OBAFEMI-AJAYI et al, 2014) \\
\hline 1 & $\operatorname{tr}$ & & \\
\hline 2 & ft esquerdo & $\mathbf{X}$ & $\mathbf{X}$ \\
\hline 3 & ft direito & $\mathbf{X}$ & $\mathbf{X}$ \\
\hline 4 & $\mathrm{~g}$ & $\mathbf{X}$ & $\mathbf{X}$ \\
\hline 5 & $\mathrm{n}$ & & \\
\hline 6 & zy esquerdo & & \\
\hline 7 & zy direito & & \\
\hline 8 & ex esquerdo & $\mathbf{X}$ & $\mathbf{X}$ \\
\hline 9 & ex direito & $\mathbf{X}$ & $\mathbf{X}$ \\
\hline 10 & p esquerdo & & $\mathbf{X}$ \\
\hline 11 & $\mathrm{p}$ direito & & $\bar{X}$ \\
\hline 12 & en esquerdo & $\mathbf{X}$ & $\mathbf{X}$ \\
\hline 13 & en direito & $\mathbf{X}$ & $\mathbf{X}$ \\
\hline 14 & mf esquerdo & & \\
\hline 15 & $\mathrm{mf}$ direito & & \\
\hline 16 & se & $\mathbf{X}$ & $\mathbf{X}$ \\
\hline 17 & al esquerdo & $\mathbf{X}$ & $\mathbf{X}$ \\
\hline 18 & al direito & $\mathbf{X}$ & $\mathbf{X}$ \\
\hline 19 & sn & $\mathbf{X}$ & $\mathbf{X}$ \\
\hline 20 & go esquerdo & & \\
\hline 21 & go direito & & \\
\hline 22 & ch esquerdo & $\mathbf{X}$ & $\mathbf{X}$ \\
\hline 23 & ch direito & $\mathbf{X}$ & $\mathbf{X}$ \\
\hline 24 & cph esquerdo & $\mathbf{X}$ & $\mathbf{X}$ \\
\hline 25 & cph direito & $\mathbf{X}$ & $\mathbf{X}$ \\
\hline 26 & ls & $\mathbf{X}$ & $\mathbf{X}$ \\
\hline 27 & sto & & \\
\hline 28 & sl & & \\
\hline 29 & gn & $\mathbf{X}$ & $\mathbf{X}$ \\
\hline & TOTAL: & 17 & 19 \\
\hline
\end{tabular}

A detecção das regiões faciais (olhos, nariz e boca) também é feita utilizando-se classificadores Haar Cascade. Essa parte do processamento considera apenas a área da face previamente detectada. A Figura 3 mostra o resultado deste processamento, na qual cada componente é identificado por um retângulo de cor diferente. 
Uma contribuição ao auxílio do diagnóstico do autismo a partir do processamento de imagens para extração de medidas antropométricas

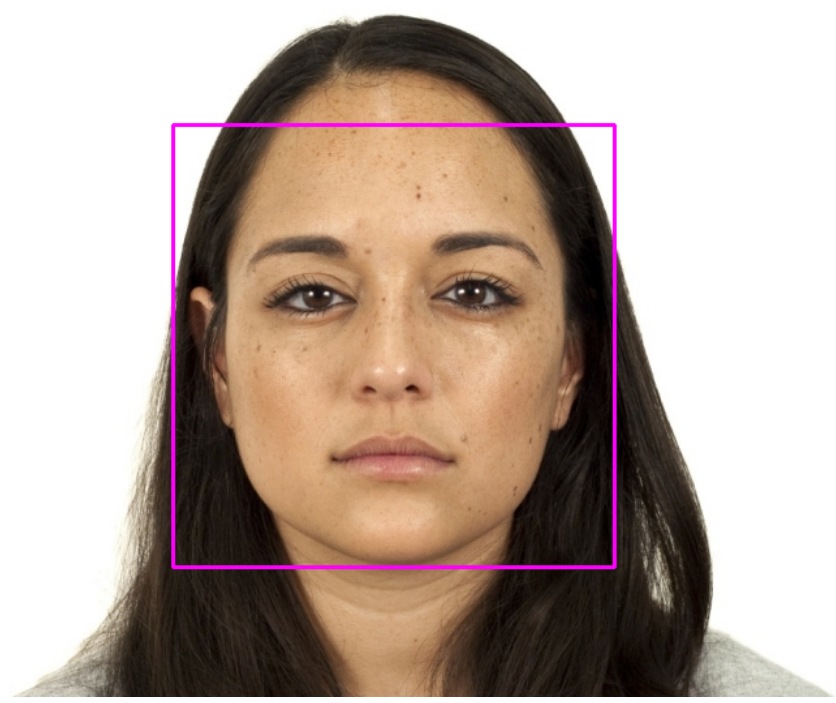

Figura 2. Detecção da face utilizando um classificador Haar Cascade.

\subsection{Pré-processamento e detecção dos landmarks}

Para cada componente facial (olhos, nariz e boca), foram aplicadas técnicas de préprocessamento de imagens a fim de preparar a imagem para identificação dos landmarks. Em seguida, são aplicadas técnicas de detecão de bordas e limiarização. Conforme mostra a Figura 4, são empregados os seguintes passos:

1. a região que será analisada é extraída da imagem original na qual foi anteriormente detectada a região da face;

2. a imagem colorida é transformada em escala de cinza;

3. para minimizar ruídos (cicatrizes, barba, pintas, manchas na pele) é aplicado o filtro Gaussian Blur [19];

4. a imagem sofre uma transformação de contraste para destacar a parte escura dos lábios. É utilizado um método da biblioteca OpenCV [18] que dobra o valor do contraste. O método recebe como argumento um valor para o canal alpha e um valor para o canal beta da imagem. O valor passado para alpha é 1 e para beta é 2. A Equação 1 mostra a tranformação feita por esse método. 
Uma contribuição ao auxílio do diagnóstico do autismo a partir do processamento de imagens para extração de medidas antropométricas

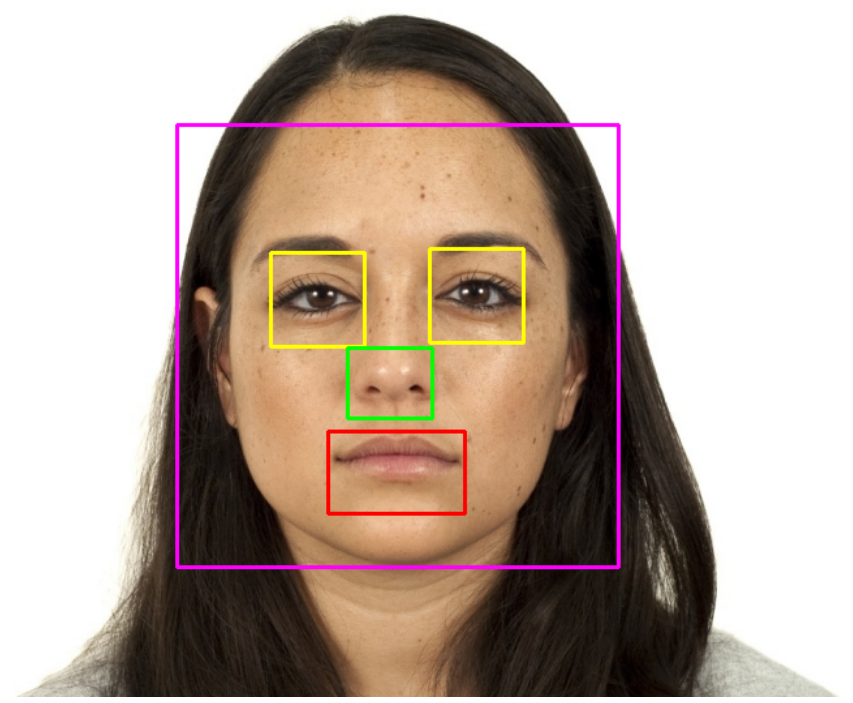

Figura 3. Detecção das regiões faciais utilizando os respectivos classificadores Haar Cascade.

$$
\text { NovaImagem }=\alpha \times \text { Imagem }+\beta
$$

Na Equação 1, NovaImagem representa a imagem após a transformação e Imagem representa a imagem antes da transformação.

5. é aplicado o filtro Canny [20] para detecção de bordas e transformação para imagem binária, usando métodos da biblioteca OpenCV [18].

6. um algoritmo percorre os pixels, localizando as coordenadas dos pontos. É feita uma iteração na imagem binária, determinando a localização dos pixels brancos (bordas). Ao término da iteração, os pixels cuja localização representa algum landmark são selecionados. Exemplo para a região da boca: (i) os dois pixels mais afastados horizontalmente, ou seja, com o menor e o maior valor de abscissa; (ii) os dois pixels com a menor coordenada de linha na borda superior e (iii) o pixel pertencente à borda superior entre os dois pixels em (ii) com a maior coordenada de linha (item (f) na Figura 5).

A Figura 5 mostra um exemplo da execução do processamento para a área da boca, desde o recorte da área de interesse da imagem da face, passando pela aplicação de transformações e filtros até a detecção dos landmarks. 
Uma contribuição ao auxílio do diagnóstico do autismo a partir do processamento de imagens para extração de medidas antropométricas

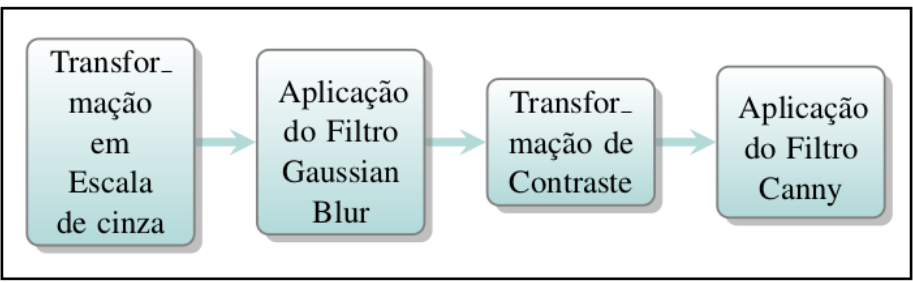

Figura 4. Passos do processamento para cálculo dos landmarks

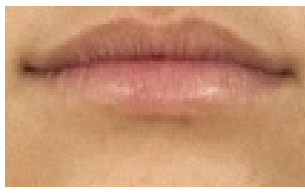

(a)

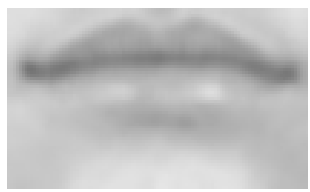

(d)

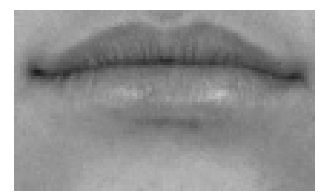

(b)

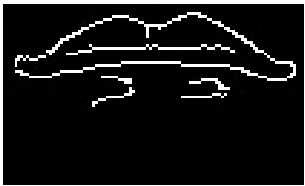

(e)

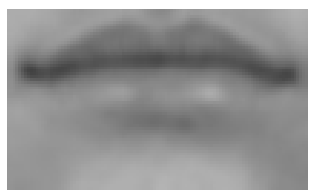

(c)

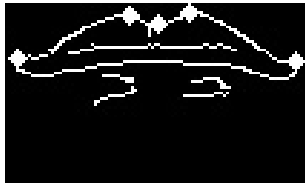

(f)

Figura 5. Localização dos landmarks da boca. (a) Recorte da boca. (b) Transformação em Escala de cinza. (c) Aplicação do Filtro Gaussian Blur. (d) Realce do brilho. (e) Aplicação do Filtro Canny. (f) Imagem binária gerada em (e) com a localização dos landmarks.

A Figura 6 ilustra a detecção dos landmarks e a representação das distâncias calculadas. Em (a) os landmarks são marcados, seguidos das respectivas nomenclaturas. Em (b), além dos landmarks, linhas brancas representam as 136 distâncias possíveis entre eles. 
Uma contribuição ao auxílio do diagnóstico do autismo a partir do processamento de imagens para extração de medidas antropométricas

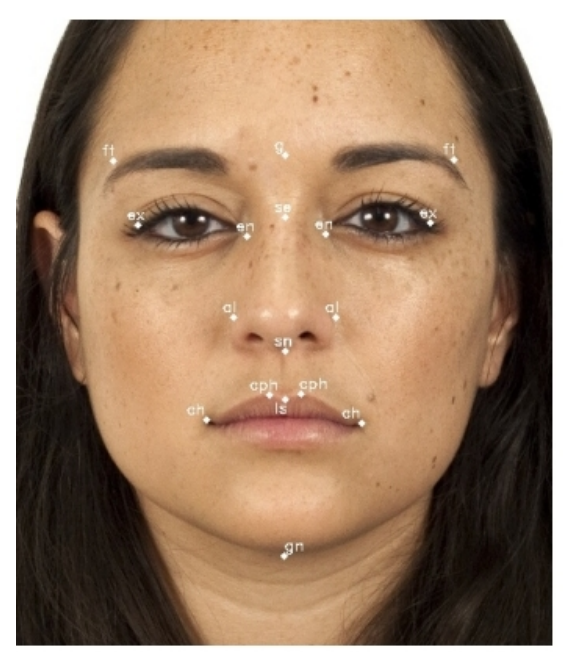

(a) Landmarks

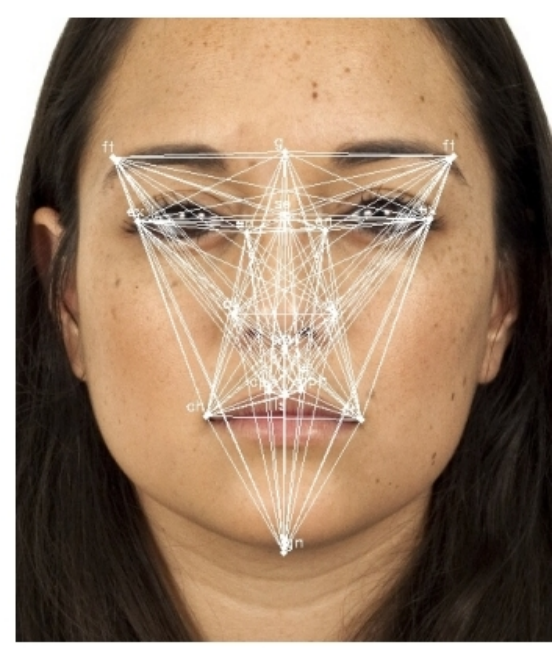

(b) Distâncias

Figura 6. Landmarks detectados e as distâncias entre eles.

\subsection{Cálculo das distâncias entre os landmarks}

As coordenadas dos landmarks foram analisadas e todas as distâncias euclidianas possíveis entre pares de landmarks apresentados na Figura 6, item (a), foram calculadas, resultando em um total de 136 medidas.

Como as imagens utilizadas possuem tamanhos e resoluções espaciais diferentes, as medidas extraídas foram normalizadas, de forma a torná-las uniformes. Para tanto, utilizouse as proporções da face, mais precisamente, os tamanhos horizontal e vertical (largura e altura), formados pelas distâncias $\mathrm{ft}_{\text {direito }}-\mathrm{ft}_{\text {esquerdo }}$ e g-gn, respectivamente, como mostra a Figura 7. Foi feita uma multiplicação entre as distâncias $\mathrm{ft}_{\text {direito }}-\mathrm{ft}_{\text {esquerdo }}$ e g-gn e todas as medidas foram divididas por esse valor, como mostra a Equação 2.

$$
\text { MedidaNormalizada }=\text { MedidaOriginal } \div\left(\left(f t_{\text {direito }}-f t_{\text {esquerdo }}\right) \times(g-g n)\right)
$$

Na Equação 2, MedidaNormalizada representa a medida com o novo valor, após a normalização; MedidaOriginal representa a medida que foi extraída da imagem, antes da normalização; $\left(f t_{\text {direito }}-f t_{\text {esquerdo }}\right)$ representa a distância entre os pontos ft dos lados direito e esquerdo da face; $(g-g n)$ representa a distância entre os pontos $\mathrm{g}$ e gn. 
Uma contribuição ao auxílio do diagnóstico do autismo a partir do processamento de imagens para extração de medidas antropométricas

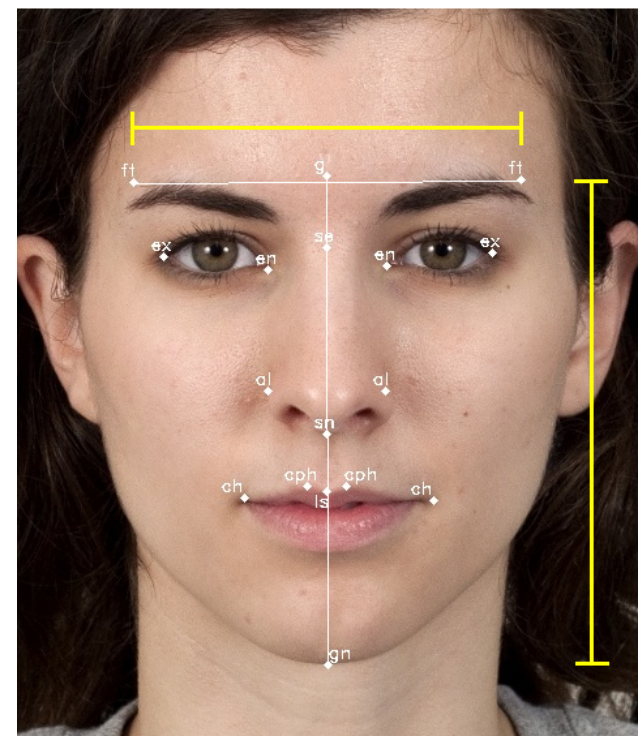

Figura 7. Tamanho da face utilizado para normalização das medidas. As linhas amarelas destacam os tamanhos horizontal e vertical da face, formados pelas distâncias $\mathrm{ft}_{d i r}-\mathrm{ft}_{e s q} \mathrm{e}$ g-gn.

Como pode-se observar, os landmarks situam-se na área delimitada pelas duas distâncias citadas. Dessa forma, as diferenças entre tamanho e resolução que existem nas imagens utilizadas são minimizadas e não interferem no resultado.

As distâncias foram calculadas em pixels e a variação entre elas está apresentada em termos percentuais na Seção 5. O cálculo das distâncias foi feito sem aproximações decimais e somente a porcentagem final foi aproximada para duas casas decimais. Considerando a variação da resolução espacial das imagens - entre 72 e 1200 dpi (dots per inch) - e a formulação da distância Euclidiana, a incerteza das medidas obtidas é menor que um pixel. As comparações foram tecidas em termos percentuais, diminuindo a influência da incerteza nos resultados.

A saída do processamento de cada imagem é um arquivo no formato CSV [21], contendo os pontos que formam as distâncias (exemplo: g-gn, significando a distância do ponto $\mathrm{g}$ ao ponto gn) seguidos dos valores das distâncias. Esses arquivos serviram de entrada para a elaboração de uma planilha eletrônica, por meio da qual os dados foram comparados. 
Uma contribuição ao auxílio do diagnóstico do autismo a partir do processamento de imagens para extração de medidas antropométricas

\subsection{Experimento conduzido}

As técnicas que compuseram a ferramenta computacional foram validadas por meio do processamento de imagens frontais de indivíduos com e sem TEA. Foram calculadas as 136 distâncias (variáveis independentes) entre todos os landmarks para cada imagem. Cada variável independente é a distância Euclidiana entre um par de landmarks, conforme apresentado na Figura 6. Foram extraídas medidas de 25 imagens de indivíduos sem TEA e 19 imagens de indivíduos com TEA.

Em seguida, foi calculada a média e o desvio padrão de cada uma das variáveis independentes (distâncias euclidianas). Os resultados foram analisados e comparados aos resultados dos trabalhos citados na literatura.

Os dois grupos usados para validação foram formados da seguinte maneira:

- Grupo 1: grupo de controle, constituído por 25 imagens de indivíduos adultos, de ambos os sexos, sem TEA, com idades variadas, obtidas de uma base de dados pública $[22]$

- Grupo 2: grupo de indivíduos com TEA, formado por 19 imagens de pacientes com idade entre 8 e 16 anos, de ambos os sexos, obtidas do Instituto de Psiquiatria da Universidade de São Paulo e da Universidade Federal de São Paulo.

\section{Resultados e Discussão}

As imagens dos grupos citados na seção anterior foram processadas em dois experimentos. No primeiro, todas as imagens foram consideradas. Em seguida, as imagens foram submetidas a uma série de avaliações em relação a alinhamento e posicionamento, assim como em relação à influência do gênero nas medidas obtidas. Assim, em um segundo experimento, algumas imagens foram desconsideradas, conforme apresentado a seguir.

Os resultados obtidos no primeiro experimento, após o processamento considerando todas as imagens da base descrita na seção anterior, mostraram que todas as médias das 136 distâncias no Grupo 2 são maiores se comparadas às do Grupo 1. Em média, as distâncias no grupo com TEA são 8,53\% maiores - média geral -, com um desvio-padrão de 7,22.

A Tabela 2 mostra todas as distâncias que apresentaram, no Grupo 2, aumento maior em porcentagem que a média geral de aumento nas medidas obtidas no mesmo grupo. Dentre as 136 distâncias, $61(44,85 \%)$ tiveram aumento maior que a média. Nesta tabela, alguns landmarks possuem as terminações “_esq" e “_dir”, que representam, respectivamente, os lados esquerdo e direito da face. 
Uma contribuição ao auxílio do diagnóstico do autismo a partir do processamento de imagens para extração de medidas antropométricas

Tabela 2. Distâncias com aumentos maiores que a média geral.

\begin{tabular}{|c|c|c|c|}
\hline Distâncias & Médias - aumento (\%) & Distâncias & Médias - aumento (\%) \\
\hline se-en_esq & 15,75 & sn-ch_esq & 9,41 \\
\hline cph_dir-gn & 14,43 & ft_dir-gn & 9,38 \\
\hline 1s-gn & 14 & al_esq-se & 9,37 \\
\hline gn-cph_esq & 13,96 & ft_dir-al_dir & 9,33 \\
\hline cph_dir-ch_dir & 13,93 & 1s-ch_esq & 9,32 \\
\hline ex_dir-en_dir & 13,29 & al_esq-ch_esq & 9,32 \\
\hline cph_esq-ch_esq & 12,81 & gn-se & 9,27 \\
\hline ex_dir-en_esq & 11,94 & ex_esq-ch_esq & 9,27 \\
\hline ch_dir-sn & 11,85 & ex_dir-ex_esq & 9,24 \\
\hline g-en_esq & 11,67 & ch_dir-en_esq & 9,16 \\
\hline gn-sn & 11,33 & ch_dir-se & 9,15 \\
\hline ex_dir-al_dir & 11,01 & ft_dir-ch_dir & 9,14 \\
\hline ft_dir-en_esq & 10,96 & g-gn & 9,05 \\
\hline ft_dir-en_dir & 10,91 & ch_dir-cph_esq & 9,03 \\
\hline ex_dir-se & 10,76 & en_dir-g & 8,96 \\
\hline ex_dir-al_esq & 10,75 & en_dir-gn & 8,94 \\
\hline en_dir-en_esq & 10,74 & en_dir-al_esq & 8,92 \\
\hline ch_dir-al_dir & 10,43 & ch_dir-g & 8,87 \\
\hline ex_dir-g & 10,17 & ft_esq-ch_esq & 8,85 \\
\hline ch_dir-al_esq & 10,04 & ex_dir-sn & 8,83 \\
\hline ch_dir-gn & 9,95 & gn-en_esq & 8,83 \\
\hline ch_dir-ls & 9,9 & gn-ex_esq & 8,82 \\
\hline gn-al_dir & 9,82 & ft_dir-ex_esq & 8,82 \\
\hline ft_dir-g & 9,78 & ex_dir-ft_esq & 8,77 \\
\hline ex_dir-gn & 9,77 & en_dir-ch_dir & 8,76 \\
\hline gn-al_esq & 9,72 & g-al_esq & 8,65 \\
\hline en_esq-al_dir & 9,7 & gn-ft_esq & 8,6 \\
\hline ft_dir-al_esq & 9,68 & se-al_dir & 8,59 \\
\hline ex_dir-ch_dir & 9,67 & ex_dir-ch_esq & 8,56 \\
\hline al_esq-al_dir & 9,64 & al_esq-sn & 8,55 \\
\hline ft_dir-se & 9,62 & & \\
\hline
\end{tabular}

A distância com o maior aumento foi a se-en_esq (aumento de 15,75\%), que representa a distância do ponto se ("sellion, ponto na porção mais profunda do ângulo nasofrontal" [1]) ao canto interno do olho esquerdo. O menor aumento $(1,31 \%)$ foi na distância cph_esq-sn. A Figura 8 destaca as distâncias com o maior (Figura 8a) e o menor (Figura8b) aumento nas 
Uma contribuição ao auxílio do diagnóstico do autismo a partir do processamento de imagens para extração de medidas antropométricas

medidas do Grupo 2. Embora o indivíduo utilizado nesta representação pertença à amostra de indivíduos do Grupo 1, ele foi escolhido apenas para ilustrar o maior e o menor aumento nas distâncias do Grupo 2, pois, devido a questões éticas, optou-se por não divulgar os indivíduos com TEA cujas imagens foram utilizadas neste trabalho.

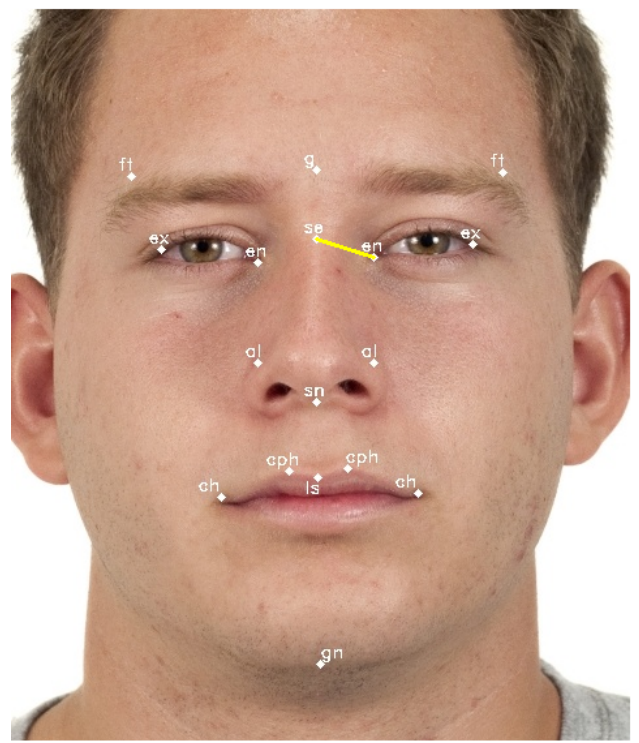

(a) Distância com o maior aumento

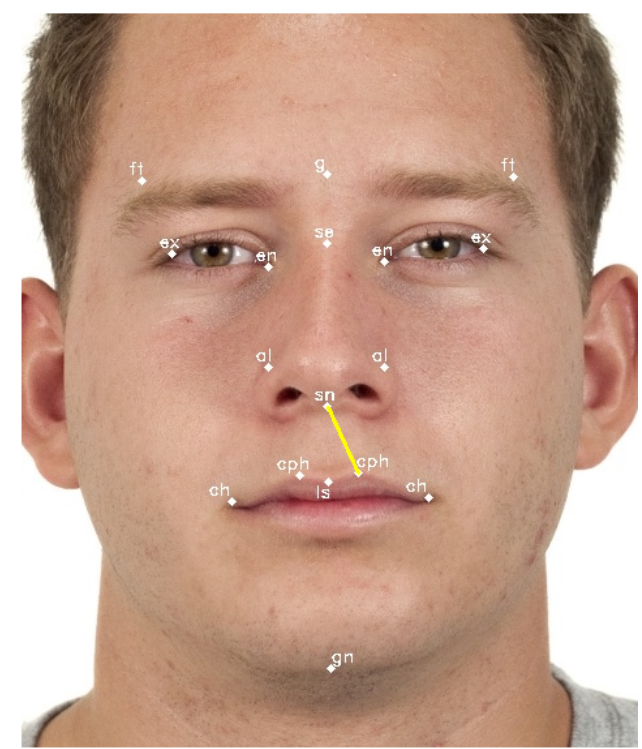

(b) Distância com o menor aumento

Figura 8. Distâncias com o maior e o menor aumento na amostra de indivíduos do Grupo 2, em relação à amostra de indivíduos do Grupo 1 (linhas amarelas). (a) Distância com o maior aumento (15,75\%; se-en_esq); (b) Distância com o menor aumento (1,31\%; cph_esq-sn).

$\mathrm{Na}$ amostra de indivíduos com TEA, havia 7 imagens fora do padrão necessário à utilização do sistema (discutido no final desta seção). Essas imagens foram removidas da amostra e os dados foram novamente analisados em um segundo experimento, ou seja, todas as medidas foram novamente calculadas, a fim de que fosse possível avaliar o quanto imagens fora do padrão poderiam ameaçar o desempenho do método apresentado. Além disso, foram considerados apenas os indivíduos do sexo masculino, para que eventuais diferenças referentes ao sexo não interferissem nos resultados. Dessa forma, comparou-se as medidas de 12 imagens remanescentes do Grupo 1 com 11 imagens remanescentes do Grupo 2 e os seguintes resultados foram encontrados: (a) no Grupo 2, as médias de 21 distâncias são menores e de 115 distâncias são maiores em relação às distâncias correspondentes no Grupo 1; (b) a média de aumento/redução nas medidas foi de $2,64 \%$, bem menor em relação aos resultados 
Uma contribuição ao auxílio do diagnóstico do autismo a partir do processamento de imagens para extração de medidas antropométricas

encontrados no primeiro experimento, que usava toda a base de imagens; (b) o desvio padrão na segunda análise foi de 12,7 e, portanto, relativamente maior ao desvio padrão encontrado no primeiro experimento (que foi de 7,22).

Das 21 distâncias na amostra do Grupo 2 cuja média foi menor em relação à amostra do Grupo 1, três $(14,28 \%)$ convergem com o trabalho de [8], isto é, as médias dessas distâncias também apresentaram-se menores no grupo com TEA em relação ao grupo sem TEA no trabalho citado. Similarmente, quatro $(19,04 \%)$ divergem, isto é, no presente trabalho as médias dessas distâncias foram menores no grupo com TEA enquanto que no trabalho de [8] essas médias apresentaram-se maiores, também no grupo com TEA.

Os números apresentados nas Tabelas 3 e 4 indicam a média da porcentagem. Valores positivos indicam o quanto (em percentual) a média do grupo com TEA foi maior que a média do grupo sem TEA. Valores negativos indicam o quanto a média do grupo com TEA foi menor que a média do grupo sem TEA. As porcentagens de aumento e redução das medidas não foram divulgadas em [8], o que inviabilizou uma comparação com os percentuais encontrados neste trabalho.

Analogamente, das 115 distâncias na amostra do Grupo 2 que tiveram um aumento em relação à amostra do Grupo $1,18(15,65 \%)$ convergem e oito $(6,95 \%)$ divergem de [8]. As Tabelas 3 e 4 mostram as medidas encontradas neste trabalho que, respectivamente, convergem ou divergem dos resultados em [8]. A Figura 9 ilustra essas medidas. Vale destacar que não foi possível comparar todas as diferenças nas medidas aqui apresentadas com as diferenças apresentadas em outros estudos. Nos estudos anteriores, apenas as distâncias que apresentaram aumentos/reduções mais expressivos foram destacadas.

Tabela 3. Medidas deste trabalho que convergem com os resultados de [8].

\begin{tabular}{|c|c|c|c|}
\hline Distância & $\mathbf{\%}$ & Distância & \% \\
\hline ls-sn & $-3,72$ & cph_dir-ch_esq & 3,86 \\
\hline se-cph_esq & $-0,97$ & cph_dir-ch_dir & 4,07 \\
\hline cph_dir-se & $-0,71$ & ft_dir-ch_esq & 4,24 \\
\hline al_esq-sn & 0,02 & ex_dir-ft_dir & 4,84 \\
\hline ch_dir-ch_esq & 0,13 & ft_esq-ex_esq & 4,91 \\
\hline cph_dir-ls & 1,39 & ls-ch_esq & 5,69 \\
\hline gn-ch_esq & 1,49 & ft_dir-al_esq & 6,27 \\
\hline ls-cph_esq & 1,55 & ft_dir-en_dir & 10,41 \\
\hline ch_dir-ft_esq & 3,28 & cph_esq-ch_esq & 11,49 \\
\hline ex_esq-en_esq & 3,33 & ex_dir-en_dir & 15,34 \\
\hline ft_esq-en_esq & 3,81 & & \\
\hline
\end{tabular}

Suspeita-se que a utilização de adultos no Grupo 1 possa ter interferido nesses re- 
Uma contribuição ao auxílio do diagnóstico do autismo a partir do processamento de imagens para extração de medidas antropométricas

Tabela 4. Medidas deste trabalho que divergem dos resultados de [8]

\begin{tabular}{|c|c|}
\hline Distâncias & \% \\
\hline cph_esq-sn & $-10,05$ \\
\hline cph_dir-cph_esq & $-4,26$ \\
\hline ch_dir-ls & $-0,74$ \\
\hline ch_dir-cph_esq & $-0,71$ \\
\hline en_dir-se & 0,35 \\
\hline ls-se & 0,65 \\
\hline g-sn & 3,28 \\
\hline en_dir-en_esq & 4,16 \\
\hline en_dir-g & 4,5 \\
\hline se-al_dir & 4,84 \\
\hline g-en_esq & 7,81 \\
\hline se-en_esq & 10,09 \\
\hline
\end{tabular}

sultados, visto que no Grupo 2, os indivíduos ainda estão em desenvolvimento e podem ter suas medidas ou proporções faciais alteradas, conforme comentam [8]. Além disso, neste trabalho foi utilizada uma análise de estatística univariada, na qual as 136 distâncias Euclidianas formam 136 variáveis independentes $(\mathrm{p}=136)$, cujo único parâmetro de comparação é a média das distâncias; isso indica um grande potencial para estudos futuros que utilizem análises matemáticas mais robustas, como técnicas de estatatística multivariada. No entanto, é importante salientar que o objetivo do experimento aqui conduzido foi avaliar a viabilidade da metodologia para encontrar os landmarks automaticamente e calcular as medidas antropométricas em qualquer tipo de imagem frontal facial, sem intervenção humana. Novos experimentos poderão ser conduzidos com outras imagens a fim de investigar a relação entre as medidas antropométricas e a presença de TEA. Por enquanto, a base de imagens utilizada colaborou para comprovar a eficiência dos métodos desenvolvidos.

Na Figura 9(a), as linhas brancas representam distâncias que se mostraram menores neste trabalho e em [8], ao passo que as linhas vermelhas representam distâncias que se mostraram menores neste trabalho, mas maiores em [8]. Já na Figura 9(b), as linhas brancas representam as distâncias que se mostraram maiores neste trabalho e em [8], enquanto que as linhas vermelhas representam distâncias que se mostraram maiores neste trabalho, mas menores em [8].

Analogamente ao apresentado na Figura 8, por questões éticas o indivíduo da Figura 9 pertence à amostra de indivíduos do Grupo 1, mas foi utilizado apenas para ilustrar as distâncias coincidentes e divergentes com [8].

Comparando-se os resultados deste trabalho com os resultados de estudos anteriores 
Uma contribuição ao auxílio do diagnóstico do autismo a partir do processamento de imagens para extração de medidas antropométricas

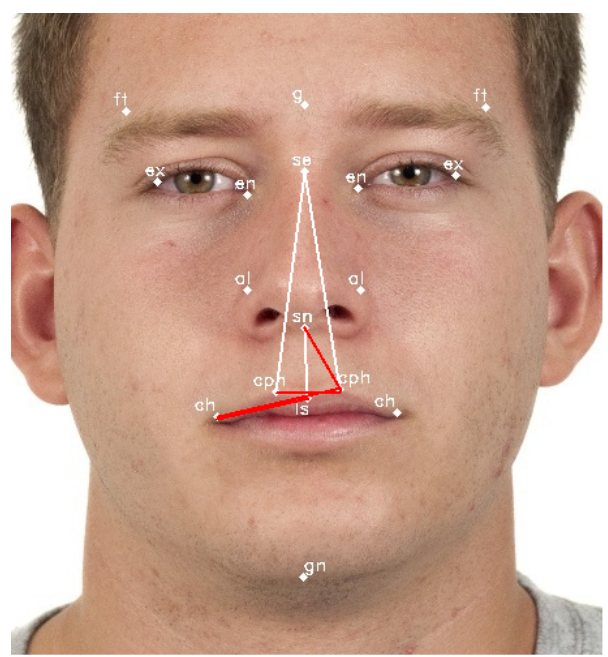

(a) Medidas com redução no Grupo 2

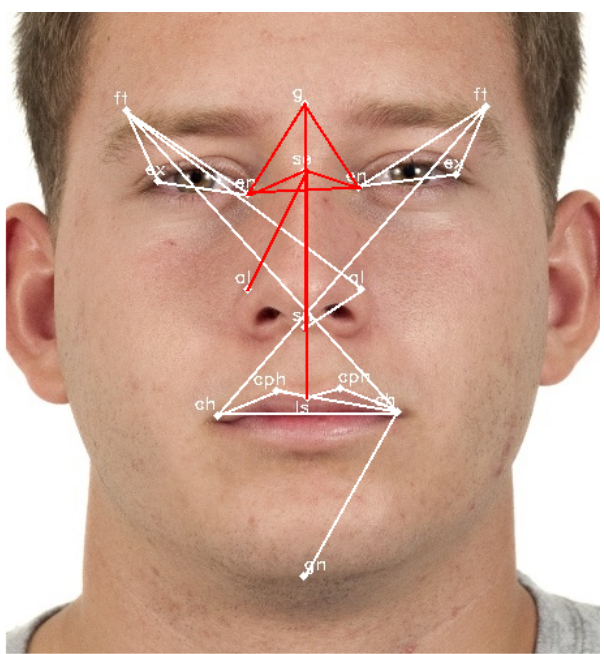

(b) Medidas com aumento no Grupo 2

Figura 9. Distâncias que apresentaram diferenças entre o Grupo 1 e o Grupo 2: (a) distâncias que apresentaram redução no Grupo 2; (b) distâncias que apresentaram aumento no Grupo 2.

que utilizaram uma representação tridimensional das faces estudadas, calculando distâncias Euclidianas [8] e geodésicas [16], percebe-se que a utilização de imagens bidimensionais reduz a precisão na extração e, consequentemente, no cálculo das medidas antropométricas. Nessas imagens não é possível determinar a profundidade das regiões faciais, como o queixo inclinado para a frente ou os olhos retraídos, por exemplo. Mesmo assim, a abordagem adotada neste trabalho mostrou-se válida, dada a convergência de várias medidas, como citado anteriormente. Adicionalmente, verifica-se que a presente abordagem tem a vantagem de permitir aquisição da imagem com dispositivos simples e de baixo custo, o que favorece a utilização da ferramenta construída na rotina clínica.

A partir dos resultados obtidos, verifica-se que a utilização de imagens fora do padrão influencia diretamente nas medidas extraídas pelo sistema. Para a correta extração das medidas antropométricas, as imagens devem ser frontais, sem sorriso, sem cabelos cobrindo a testa e não podem ser utilizadas imagens de perfil. A Figura 10 mostra o tipo de imagem correto e os tipos de imagem que não devem ser utilizados. Na Figura 10(a) é apresentada uma imagem que resulta em processamento correto, com a face totalmente voltada para frente e sem nenhuma angulação. Na Figura 10(b), a face está voltada para o lado esquerdo e na 
Uma contribuição ao auxílio do diagnóstico do autismo a partir do processamento de imagens para extração de medidas antropométricas

Figura 10(c) a imagem foi obtida com o indivíduo de perfil. Estes dois últimos tipos de imagens são inadequados para o propósito deste trabalho e podem influenciar nos resultados caso estejam presentes na base de imagens. Salienta-se, no entanto, que é possível obter imagens adequadas com equipamentos simples e de baixo custo, como já citado anteriormente, o que corrobora com a viabilidade da proposta aqui apresentada.

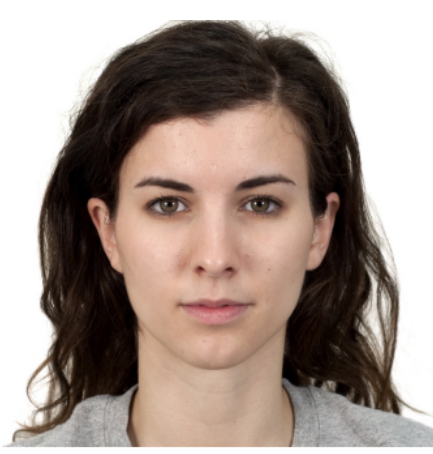

(a) Imagem ideal

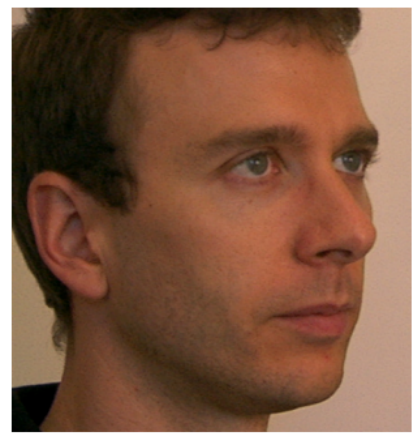

(b) Indivíduo olhando para um dos lados

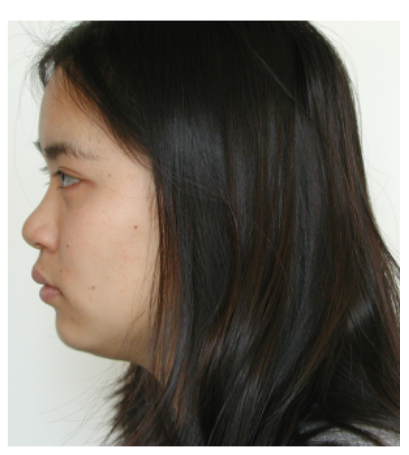

(c) Imagem de perfil

Figura 10. Tipos de imagens. (a) Imagem frontal, adequada para o processamento da presente proposta. (b) e (c) Imagens parcialmente ou totalmente de perfil [23], inadequadas para processamento com a abordagem proposta.

Notou-se que em algumas imagens o sistema não conseguiu detectar a face e em outras, após detectada a face, não foi possível detectar alguma região facial (olhos, nariz ou boca). Em geral, essas falhas ocorrem quando as imagens apresentam luminosidade baixa ou baixo contraste entre os componentes a serem extraídos e a pele do indivíduo. Apesar desta limitação, em uma aplicação da ferramenta na rotina clínica, é possível melhorá-la para evitar tais falhas. Uma abordagem possível é permitir o processamento dos componentes em tempo real imediatamente após a aquisição da imagem, permitindo o descarte de imagens inadequadas e a obtenção de novas amostras, se necessário.

Embora o sistema tenha conseguido extrair medidas de imagens em diversos formatos, tamanhos e resoluções, percebeu-se que imagens com tamanho aproximado de 1200 x 800 e resolução de $72 \times 72$ pixels por polegada obtiveram melhores resultados, ou seja, menos falhas na detecção dos landmarks. 
Uma contribuição ao auxílio do diagnóstico do autismo a partir do processamento de imagens para extração de medidas antropométricas

\section{Conclusão}

Este trabalho propôs a aplicação de técnicas de processamento de imagens com o intuito de extrair medidas antropométricas. As técnicas foram expostas e mostraram-se eficientes para a extração dessas medidas.

As técnicas empregadas neste trabalho são capazes de detectar os principais landmarks faciais e calcular as distâncias entre eles. Estima-se que com uma base de imagens maior e mais uniforme os resultados sejam mais próximos dos resultados de estudos anteriores ([8], [16]). No entanto, é importante salientar que o objetivo dos experimentos conduzidos no presente trabalho era validar as técnicas propostas. Não era pretensão do presente trabalho obter resultados conclusivos sobre a relação ente medidas antropométricas faciais e a presença de TEA em indivíduos. Após adequação da ferramenta para a aplicação na rotina médica, novos experimentos deverão ser conduzidos com a finalidade de comprovar a eficácia da ferramenta como instrumento de auxílio ao diagnóstico e, assim, facilitar a verificação da relação existente entre medidas antropométricas e presença de TEA.

Imagens fora do padrão apresentado na seção 5 geram medidas que distorcem os resultados da amostra a qual pertencem. Trabalhos futuros podem adicionar a detecção de pontos de imagens de perfil e combinar as coordenadas desses pontos com os da imagem frontal, aumentando a exatidão na detecção dos landmarks e, consequentemente, gerando medidas antropométricas mais precisas.

Como anteriormente mencionado, neste trabalho foi utilizada uma análise estatística univariada. Além de novos testes com mais amostras, trabalhos futuros são possíveis no sentido de aprofundar a análise aqui tecida, considerando, por exemplo, análise estatística multivariada, análise de componentes principais para indicar as medidas mais significativas para o diagnóstico, além de técnicas de reconhecimento de padrões que podem auxiliar na classificação de indivíduos com e sem TEA.

Representando cada indivídio por um vetor de características 136-dimensional contendo as 136 distâncias obtidas, técnicas de aprendizado supervisionado podem ser aplicadas a fim de selecionar as características mais relevantes e assim construir classificadores, como por exemplo os baseados em Máquinas de Vetores de Suporte (SVM), para a discriminação entre casos e controle.

A inexistência de bases de imagens públicas é um desafio a ser vencido para validar mais adequadamente a proposta do presente trabalho. Por isso, também está prevista como trabalho futuro a formação de uma base de imagens, robusta e com casos variados. Tal base conterá casos com variação de faixa etária, etnias e gênero, a fim de verificar se há diferenças das medidas extraídas quando tais variáveis são consideradas. De posse de mais dados, será possível também aplicar técnicas de aprendizado não-supervisionado no grupo de indivíduos 
Uma contribuição ao auxílio do diagnóstico do autismo a partir do processamento de imagens para extração de medidas antropométricas

acometidos com o TEA a fim de identificar agrupamentos que possam estar associados a subgrupos fenotípicos do transtorno.

O método desenvolvido no presente trabalho que culminou na implementação de uma ferramenta computacional mostrou-se robusto, capaz de medir as distâncias faciais e fornecer dados para comparar tais distâncias entre grupos com e sem TEA, em imagens com diferentes resoluções espaciais e de contraste. Embora algumas melhorias e testes mais exaustivos devam ser conduzidos para que seja possível sua aplicação clínica, a ferramenta é uma contribuição efetiva para que a extração e a comparação de medidas antropométricas faciais possam ser utilizadas, de forma prática e rápida, para auxiliar o diagnóstico do TEA.

\section{Agradecimentos}

Deixamos expressos nossos sinceros agradecimentos a Andressa Zambori e Débora Zambori, do Hospital das Clínicas de São Paulo, pela receptividade, acolhida, apoio administrativo e ajuda na obtenção das imagens de alguns pacientes.

\section{Contribuição dos Autores:}

Os autores contribuíram igualmente na realização deste trabalho.

\section{Referências}

1 MELlO, A. M. S. R. Autismo: guia prático. 4. ed. [S.1.]: São Paulo: AMA; Brasília: CORDE, 2005.

2 Camargos Jr, W. et al. Transtornos invasivos do desenvolvimento: $3^{o}$ Milênio. [S.1.]:

Brasília: CORDE, 2005.

3 JORGE, L. M. Instrumentos de avaliação de autistas : revisão de literatura. Campinas, SP: [s.n.], 2003.

4 GADIA, C. A.; TUCHMAN, R.; ROTTA, N. T. Autismo e doenças invasivas de desenvolvimento. Jornal de Pediatria, v. 80, n. 2, p. 83-94, 2004.

5 HASHEMI, J. et al. Computer vision tools for low-cost and noninvasive measurement of autism-related behaviors in infants. Autism Research and Treatment, v. 2014, 2014.

6 FARKAS, L. Anthropometry of the head and face. [S.1.]: Raven Press, 1994. ISBN 9780781701594. 
Uma contribuição ao auxílio do diagnóstico do autismo a partir do processamento de imagens para extração de medidas antropométricas

7 MEGUID, N. A. et al. Minor physical anomalies in adults with autism spectrum disorder and healthy controls. World Journal of Pediatrics, v. 10, n. 4, p. 318-323, 2014.

8 ALDRIDGE, K. et al. Facial phenotypes in subgroups of prepubertal boys with autism spectrum disorders are correlated with clinical phenotypes. Molecular Autism, v. 2, n. 15, 2011.

9 MANOUILENKO, I. et al. Minor physical anomalies in adults with autism spectrum disorder and healthy controls. Autism Research and Treatment, v. 2014, 2014.

10 GONZALEZ, R. C.; WOODS, R. E. Digital image processing. 2. ed. [S.1.]: Prentice-Hall, 2002.

11 ALVES, A. P. P. P. Análise comparativa da antropometria facial entre pais de crianças portadoras de fissuras labiopalatinas e indivíduos sem história familiar de fissuras. São Paulo, SP: [s.n.], 2008.

12 PRIETO, F. Antropometria facial a partir de multiples vistas: una propuesta metodologica. 2008.

13 GUEDES, D. P. Procedimentos clínicos utilizados para análise da composição corporal. Revista Brasileira de Cineantropometria e desempenho humano, v. 15, n. 1, p. 113-129, 2012.

14 RAMIRES, R. R. et al. Medidas faciais antropométricas de adultos segundo tipo facial e sexo. Revista CEFAC, v. 13, n. 2, p. 245-252, 2011.

15 RODIER, P. M.; BRYSON, S. E.; WELCH, J. P. Minor malformations and physical measurements in autism: data from new scotia. Teratology, n. 55, p. 319-325, 1997.

16 OBAFEMI-AJAYI, T. et al. Facial structure analysis separates autism spectrum disorders into meaningful clinical subgroups. Journal of Autism and Developmental Disorders, n. 45, p. 1302-1317, 2014.

17 VIOLA, P.; JONES, M. Rapid object detection using a boosted cascade of simple features. IEEE. Computer Vision and Pattern Recognition, 2001. CVPR 2001. Proceedings of the 2001 IEEE Computer Society Conference on, v. 1, n. 5, 2001.

18 HARVEY, A. Opencv face detection: Visualized. 2011.

19 CHEN, F.; MA, J. An empirical identification method of gaussian blur parameter for image deblurring. IEEE Transactions on Signal Processing, v. 57, n. 7, 2009.

20 CANNY, J. A computational approach to edge detection. IEEE Trans. on Pattern Analysis and Machine Intelligence, n. 8, p. 679-698, 1986. 
Uma contribuição ao auxílio do diagnóstico do autismo a partir do processamento de imagens para extração de medidas antropométricas

21 SHAFRANOVICH, Y. Common format and mime type for comma-separated values (csv) files. 10 2005. RFC 4180.

22 MA, D. S.; CORRELL, J.; WITTENBRINK, B. The chicago face database: A free stimulus set of faces and norming data. Behavior Research Methods, n. 47, p. 1122-1135, 2015.

23 ALVIRA, M.; RIFKIN, R. An Empirical Comparison of SNoW and SVMs for Face Detection. Cambridge, MA, 2001. 